\begin{tabular}{|c|c|c|}
\hline & Int.J.Curr.Microbiol.App.Sci (2021) 10(09): 301-313 & \multirow{4}{*}{ 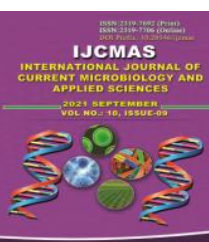 } \\
\hline & \multirow{4}{*}{$\begin{array}{l}\text { International Journal of Current Microbiology and Applied Sciences } \\
\text { ISSN: 2319-7706 Volume } 10 \text { Number } 09 \text { (2021) } \\
\text { Journal homepage: http://www.ijcmas.com }\end{array}$} & \\
\hline & & \\
\hline EXCELLENT & & \\
\hline PUBLISHERS & & www.ijemas.com \\
\hline
\end{tabular}

\title{
A Clinical-Bacteriological Profile of Multidrug Resistant Pseudomonas aeruginosa at Tertiary Care Hospital Shahjahanpur, Uttar Pradesh
}

\author{
Jitendra Kumar Chaudhary*
}

Department of Microbiology, Associate Professor, VarunArjun Medical College and
Rohilkhand Hospital, Banthra, Shahjahanpur, Pin-242307, Uttar Pradesh, India

*Corresponding author

\section{A B S T R A C T}

Keywords

Pseudomonas aeruginosa,

multidrug resistance, antibiotics, antibiogram

Article Info

Accepted:

15 August 2021

Available Online:

10 September 2021
The Pseudomonas aeruginosa is predominant agent causing nosocomial infections. In recent time, it develops resistance continuously to the antibiotics becomes Multidrugresistant $P$. aeruginosa. So, in cystic fibrosis patents it difficult to eradicate $P$. aeruginosa infections with antimicrobial treatment. Therefore, focus on alternative mechanisms for treating $P$. aeruginosa infections. On the basis of growth, morphological and biochemical characteristics, $P$. aeruginosa strains were isolated from the clinical samples in this work. After that the antibiotic sensitivity was performed and the Multidrug resistant Pseudomonas aeruginosa was identified according to CLSI standard guideline chart by measuring the zone of inhibition for $P$. aeruginosa. The isolated strains showed resistance against three or more antibiotics, considered as MDR Pseudomonas aeruginosa. By antibiogram pattern 51 showed Multi drug resistant strains out of 102 isolated strains. As P. aeruginosa abide to develop resistance to the antibiotics, the quorum sensing increased transcriptional regulator QscR might performs another target. Thus the prevalence of MDR strains of Pseudomonas aeruginosa was investigated on current study. The antibiogram pattern revealed 51 MDR strains of $P$. aeruginosa. In which the majority of strains exhibited resistance towards Piperacillin (98\%), Ciprofloxacin (90\%), Ofloxacin (90\%), Levofloxacin (80\%) and Tobramycin (60\%).

\section{Introduction}

The Pseudomonas aeruginosa is the most important agent causing nosocomial infections. It is causes predominantly bacteremia, meningitis, pneumonia and urinary tract infection. It is more common in patients with neutropenia, burns, ventilators and cystic fibrosis. ${ }^{1}$ It is continuously raising human pathogen, accountable for $12 \%$ of urinary tract infections, $10 \%$ of blood stream infections, and $8 \%$ of surgical wound infections. ${ }^{2}$ In incubated patients, it causes mostly ventilator-associated pneumonia which directly responsible to death of patients. ${ }^{3}$ P.aeruginosa infection becomes chronic infection in cystic fibrosis patients, which is highly illness and death of this 
population. Pseudomonas have ability to invade a potential host that monitors cell density through a mechanism which control virulence factors and communicate between bacteria. $^{4,5}$ Cystic fibrosis is more vulnerable nowadays because recent antibacterial treatments are unable to eradicate multidrugresistant $P$. aeruginosa infection which is more prevalent. Thus, in many research focused on alternate mechanisms to target the $P$. aeruginosa. ${ }^{6}$ According to therapeutics that target inhibit QS in $P$. aeruginosa might diminish the virulence of the bacterium and that probably help to clear the infection by host immune system. There are Two QS networks in which LasR and RhIR is ideal target in therapeutics. ${ }^{7}$

The Las system made up of LasR transcriptional regulator and LasI synthase protein. LasI synthase is necessary for the producing of the AHL signal molecule N-(3oxododecanoyl)-1-homoserine lactone (3OC12-HSL). ${ }^{8,9}$ LasR needs3O-C12-HSL for activation of LasR transcription factor.

It was recently denoted that, LasR forms multimers with 3O-C12-HSL, and only that multimeric form of LasR protein is capable to bind DNA and control the transcription of multiple genes. The second QS system to $P$. aeruginosa contains of the RhlI and RhlR proteins. ${ }^{10,11}$ The RhlI synthase is essential for producing the AHL N-butyryl-l-homoserine lactone (C4-HSL), and RhlR is transcriptional regulator. When RhlR is combined with $\mathrm{C} 4-$ HSL this controls the expression of several genes only. This has been determined that both 30-C12-HSL and C4-HSL are freely diffuse out from bacterial cells. But, 3O-C12HSL diffusion is comparatively slower than that of C4-HSL.

In adding to LasR and RhlR, there is also third Las system, orphan LasR-RhlR homolog, QscR, but which have no cognate acyl-HSL synthase gene. ${ }^{12}$ The qscR mutant is hyper virulent. It is observed that expression of some genes controlled by the LasR-I and RhlR-I that stimulus of QscR and are prematurely triggered in a qscR mutant and enclose genes the phz1 and phz2 phenazine synthesis operons; henAB, the hydrogen cyanide synthesis operon; las $\mathrm{B}$, that codes for elastase; rhlI; and lasI. The mechanism for temporary suppression of these genes by QscR is not clear. ${ }^{13,15}$ When acyl-HSL concentrations are low, the QscR might create heterodimers with LasR and RhlR. This would inactivate LasR and RhlR.

It is a greater alarming public health has aroused due to appearance and spread of multidrug resistance bacteria.

For therapeutic options, Multidrug resistant strains have very restricted option for treatment because antibiotic resistance of P.aeruginosa infection frequently complicated so need urgency for identification of novel antimicrobial targets and evolution of new antimicrobial drugs for better treatment. ${ }^{16}$ P.aeruginosa can resistance to broad-spectrum antibiotics quickly because it is omnipresent micro-organism. MDR strains of $P$. aeruginosa are an increasing cause of morbidity and mortality in burn case.

In developing nations $P$. aeruginosa causes remarkable trouble among hospitalized patients because emergence of MDR strains frequently. ${ }^{17,18}$ So, prime focus of researchers and practitioners worldwide are to identify MDR strains and contributing mechanism to give it resistance because there is limited number of studies has been done to discover MDR $P$. aeruginosa in India. Thus in the present study, Pseudomonas aeruginosa was identified and isolated from the clinical samples taken from tertiary care hospital and the Multidrug resistant strains were assessed by antibiogram pattern. 


\section{Materials and Methods}

\section{Sampling and processing}

The Clinical samples were collected in clinical hospital laboratory during March 2019 to February 2020 and each sample was taken in Peptone water containing tubes and then sent to the Microbiology laboratory. Then, the samples were streaked on Nutrient agar plates and incubated at $37^{\circ} \mathrm{C}$ for 24 hours. After 24 hours, the separate characteristic colonies were subjected to Gram's staining and then also it should sub-culture on MacConkey $\operatorname{agar}^{19}$. The pure isolated Pseudomonas aeruginosa were stored in the refrigerator at $4^{\circ} \mathrm{C}$.

\section{Identification of $P$. aeruginosa}

The sub-culture isolates in MacConkey media were identified on the basis of Bergey's manual of determinative bacteriology, ${ }^{20}$ the characterize identification by Gram staining, catalase, oxidase and motility by Hanging drop method and further by performing various biochemical tests such as sugar fermentation tests for glucose, sucrose, lactose, maltose and mannitol, Triple Sugar Iron agar test, Indole production test, citrate test, urease test, methyl red test, VogesProskauer test, Nitrate reduction test, $\mathrm{H}_{2} \mathrm{~S}$ production and Inulin test.

\section{Antibiotic Susceptibility}

Antibiotic susceptibility was performed with disc diffusion or Kirby-Bauer test method. ${ }^{21}$ The commercially available standard antibiotic discs were used for the Antibiotics sensitivity pattern identification. From subculture some bacterial colonies were inoculated into Nutrient broth and incubated at $37^{\circ} \mathrm{C}$ for $2-4$ hours and then the turbidity was adjusted to $0.5 \mathrm{McFarland}$ standards. After that, sterilized Muller Hinton agar media was prepared and solidify on petridishes then incubated up to 30 minute in incubator for remove the excess moisture from the surface and then inoculated by cotton swabs uniformly over the surface of MHA media. Antimicrobial antibodies susceptibility of each isolate was assessed by disk diffusion method i.e., Piperacillin (100g), Piperacillintazobactam $(100 / 10 \mathrm{~g})$, Tobramycin $(10 \mathrm{~g})$, Meropenem (10g), Imipenem (10g), Ceftazidine (30g), Cefepime (30g) Ofloxacin (5g), Ciprofloxacin (5g), Levofloxacin $(5 \mathrm{~g})$ and Norfloxacin $(10 \mathrm{~g})$.

The antibiotic discs were thoroughly placed on the surface of petri-dishes by sterile forceps and kept for 30 minutes at refrigerator to diffuse antibiotic in the media and then incubated at $37^{\circ} \mathrm{C}$ for 24 hours. The diameter of the clear zones of inhibition was measured by using zone measuring ruler and recorded; the measurement was according to the standard tables given CLSI.

\section{Multidrug Resistant Strains}

According to CLSI guideline chart, Multidrug resistant Pseudomonas aeruginosa was identified by measuring the zone of inhibition for Pseudomonas aeruginosa. ${ }^{22}$ The isolated strains that showed resistance against three or more antibiotics, considered as MDR Pseudomonas aeruginosa.

\section{Results and Discussion}

Pseudomonas aeruginosa is a most important agent causing nosocomial infections associated mainly with neutropenia, burns, ventilators and cystic fibrosis. Recent time, it is challenging for clinician to treat $P$. aeruginosa infection because there is frequently get resistant to many antibiotics which is lethal for patients lead to death. ${ }^{23}$ The multidrug resistant strains of $P$. aeruginosa develop rapidly. For controlling it, we need to 
use alternative method that inhibiting the QS in $P$. aeruginosa by enhancing the transcriptional regulator.

In the present study, total 1575 samples were collected from Clinical hospital laboratory at VAMC and RH during the year March 2019 to February 2020. On the basis of the growth characteristics on Peptone water, 525 samples $(33.33 \%)$ were observed for turbid growth with surface pellicle on Peptone water.

Out of 525 samples, 102 samples (19.43\%) were conformed as $P$. aeruginosa based on their growth characteristics [Fig. 1] and numerous biochemical tests were mentioned in [Table 1] and [Fig. 2].

The study report of 60 admitted patients at Vardhman Mahavir Medical College and Safdarjang hospital New Delhi in $2014^{24} 56 P$. aeruginosa isolated positive from 525 samples of Blood and wound swabs. Similarly, $495 P$. aeruginosa isolated from 1548 clinical samples at Mangalore in 2002. ${ }^{25}$ From numerous samples study from clinical hospital infections at a tertiary care hospital Andhra Pradesh reported $290 P$. aeruginosa isolates in $2012 .{ }^{26}$ A remarkable number of $P$. aeruginosa isolated at Bangalore in 2007 according to reference. ${ }^{7}$ Reference $^{27} \mathrm{P}$. aeruginosa reported out of 85 patients in New Delhi, India.

In the present study, the total 102 isolated $P$. aeruginosa were subjected to the antimicrobial susceptibility testing with different types of antibiotics such as penicillin, $\beta$-Lactam $/ \beta$-Lactamase

Inhibitor Combinations, aminoglycosides, carbapenems, cephems and Fluoroquinolones. According to the CLSI standard guide line chart for enterobacteriaceae, each antibiotic was isolated as resistant or intermediate or sensitive to measure the diameter of zone of inhibition against the antibiotics [Table 2]. If the diameter of the zone of inhibition of $\leq$ $14 \mathrm{~mm}$ for Piperacillin, $\leq 14$ for Piperacillintazobactam, $\leq 12 \mathrm{~mm}$ for Tobramycin, $\leq$ $15 \mathrm{~mm}$ for Meropenem, $\leq 15 \mathrm{~mm}$ for Imipenem, $\leq 14 \mathrm{~mm}$ for Ceftazidine, $\leq 14 \mathrm{~mm}$ for Cefepime, $\leq 12 \mathrm{~mm}$ for Oflaxacin and $\leq$ $15 \mathrm{~mm}$ for Ciprofloxacin, $\leq 13 \mathrm{~mm}$ for Levofloxacin, $\leq 12 \mathrm{~mm}$ for Norfloxacin then the isolate was scrutinized as resistant.

Resistance of isolated $P$. aeruginosa with three or more antibiotics was assessed as multidrug resistant strains. Out of isolated MDR, 98\% was noted resistant against Piperacillin and $75 \%$ sensitivite against Ceftazidine, Cefepime, $85 \%$ sensitivity against Pepracillin-tazobactam and 98\% sensitivity noted against Imipenem, Meropenem. The more than three antibiotics resistant isolated and their zone of inhibition pattern was exhibited in [Fig. 3]. Ramana et al., study in $2012^{26}$ they found the highest number of infections in urinary catheters, followed by endotracheal tips and central venous catheters. The antibiotic resistance was maximum incefotaxime and gentamycin (40\%) followed by ciprofloxacin (39\%), amikacin (26\%), cefoperazone- sulbactum (22\%), piperacillintazobactum (16\%), and imipenem (14\%).

The studies of Shenoy et al., in $2002{ }^{29}$ They found that $P$. aeruginosa strains were highly resistant to most anti-pseudomonal antibiotics, $68.01 \%$ sensitivity of Amikacin was most suitable for routine use and Netilmicin was most resistant antibiotic showing 70.04\% resistance but Gentamycin found a relatively higher sensitivity of $55.87 \%$. Imipenem and Meropenem was $100 \%$ sensitivity. Swetha et al., in $2017^{28}$ was isolated $19 P$. aeruginosa and studied the susceptibility against 12 antibiotics. 
Table.1 Identification of Pseudomonas aeruginosa by various Biochemical test

\begin{tabular}{|c|c|c|}
\hline S. No. & Name of the test & Observations \\
\hline $\mathbf{1}$ & Gram's staining & Gram negative rod \\
\hline $\mathbf{2}$ & Motility test & Positive \\
\hline $\mathbf{3}$ & Catalase test & Positive \\
\hline $\mathbf{4}$ & Oxidase test & Positive \\
\hline $\mathbf{5}$ & Glucose fermentation & Negative \\
\hline $\mathbf{6}$ & Sucrose fermentation & Negative \\
\hline $\mathbf{7}$ & Lactose fermentation & Negative \\
\hline $\mathbf{8}$ & Maltose fermentation & Negative \\
\hline $\mathbf{9}$ & Mannitol fermentation & Negative \\
\hline $\mathbf{1 0}$ & Acid from glucose & Positive \\
\hline $\mathbf{1 1}$ & Gas from glucose & Negative \\
\hline $\mathbf{1 2}$ & Triple sugar iron test & K/K \\
\hline $\mathbf{1 3}$ & Inulin test & Negative \\
\hline $\mathbf{1 4}$ & Indole production test & Negative \\
\hline $\mathbf{1 5}$ & Citrate utilization test & Positive \\
\hline $\mathbf{1 6}$ & Urease hydrolysis test & Negative \\
\hline $\mathbf{1 7}$ & Methyl red test & Negative \\
\hline $\mathbf{1 8}$ & Voges - Proskauer test & Negative \\
\hline $\mathbf{1 9}$ & Nitrate reduction test & Positive \\
\hline $\mathbf{2 0}$ & H2S Production & Negative \\
\hline
\end{tabular}

Table.2 CLSI guide lines table for determining the Pseudomonas aeruginosa as Resistant or Intermediate or Sensitive against various Antibiotics

\begin{tabular}{|c|c|c|c|c|}
\hline Types & Antibiotics & Resistant & Intermediate & Sensitive \\
\hline Penicillin & Piperacillin & $\leq 14$ & $15-20$ & $\geq 21$ \\
\hline $\begin{array}{c}\beta \text {-Lactam } / \beta- \\
\text { Lactamase inhibitor } \\
\text { Combinations }\end{array}$ & $\begin{array}{l}\text { Piperacillin- } \\
\text { tazobactam }\end{array}$ & $\leq 14$ & $15-20$ & $\geq 21$ \\
\hline Aminoglycosides & Tobramycin & $\leq 12$ & $13-14$ & $\geq 15$ \\
\hline \multirow[t]{2}{*}{ Carbapenems } & Meropenem & $\leq 15$ & $16-18$ & $\geq 19$ \\
\hline & Imipenem & $\leq 15$ & $16-18$ & $\geq 19$ \\
\hline \multirow[t]{2}{*}{ Cephems } & Ceftazidine & $\leq 14$ & $15-17$ & $\geq 18$ \\
\hline & Cefepime & $\leq 14$ & $15-17$ & $\geq 18$ \\
\hline \multirow[t]{4}{*}{ Fluoroquinolones } & Oflaxacin & $\leq 12$ & $13-15$ & $\geq 16$ \\
\hline & Ciprofloxacin & $\leq 15$ & $16-20$ & $\geq 21$ \\
\hline & Levofloxacin & $\leq 13$ & $14-16$ & $\geq 17$ \\
\hline & Norfloxacin & $<12$ & $13-16$ & $\geq 17$ \\
\hline
\end{tabular}


Fig.1 Culture characterization of isolated Pseudomonas aeruginosa from samples was showed as medium-sized yellowish green colored colonies. The gram' staining showed gram-negative, rod shaped and pink colored bacteria.

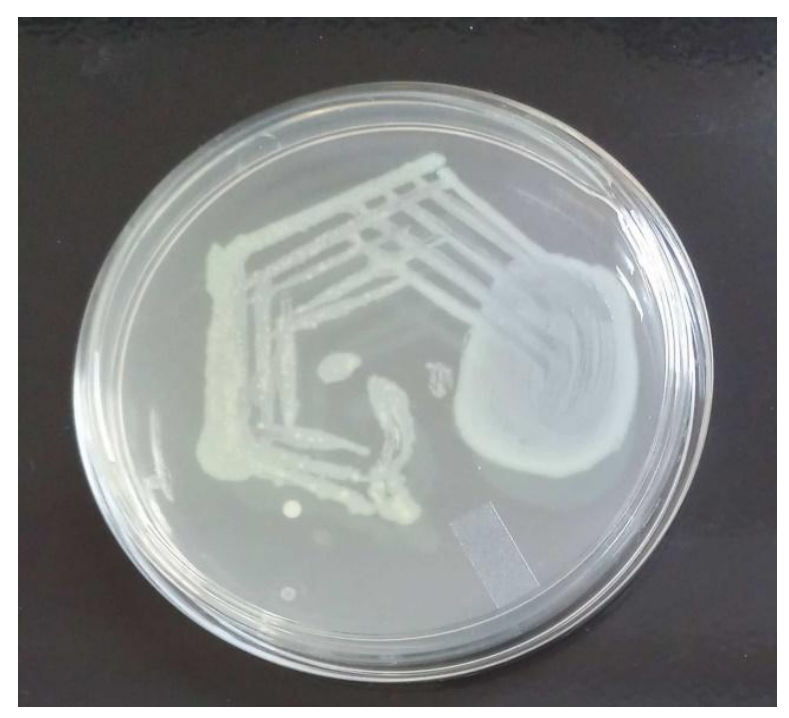

Fig.2 Biochemical behavior of Pseudomonas aeruginosa that are Sugar fermentation tests for glucose, sucrose, lactose, maltose and mannitol, Triple Sugar Iron agar test, Urease test, Citrate utilization test, Nitrate reduction test, Indole production test, methyl red test, Voges-Proskauer test, Oxidase test and Catalase test.
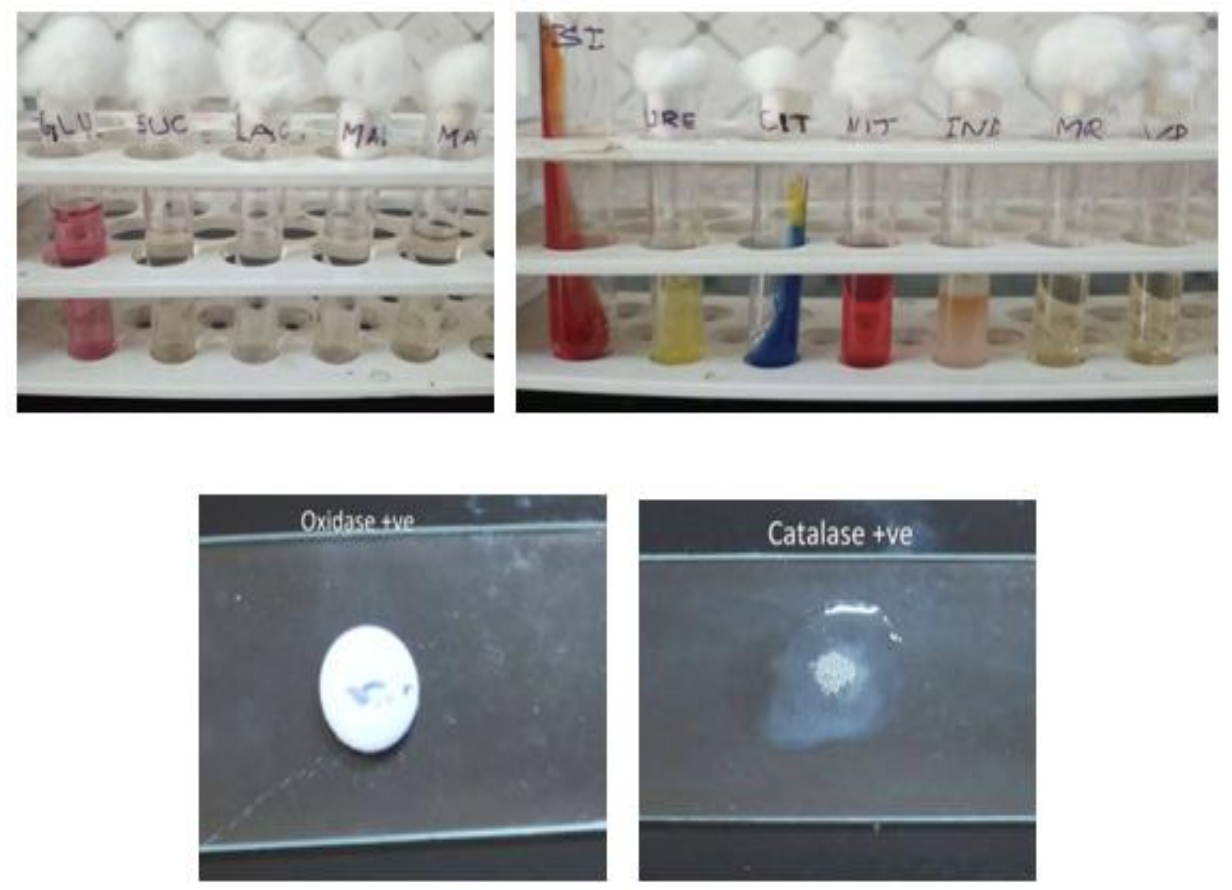
Fig.3 Antibiogram pattern of 3 isolated MDR Pseudomonas aeruginosa that are Isolate-19, Isolate-45 and Isolate-60

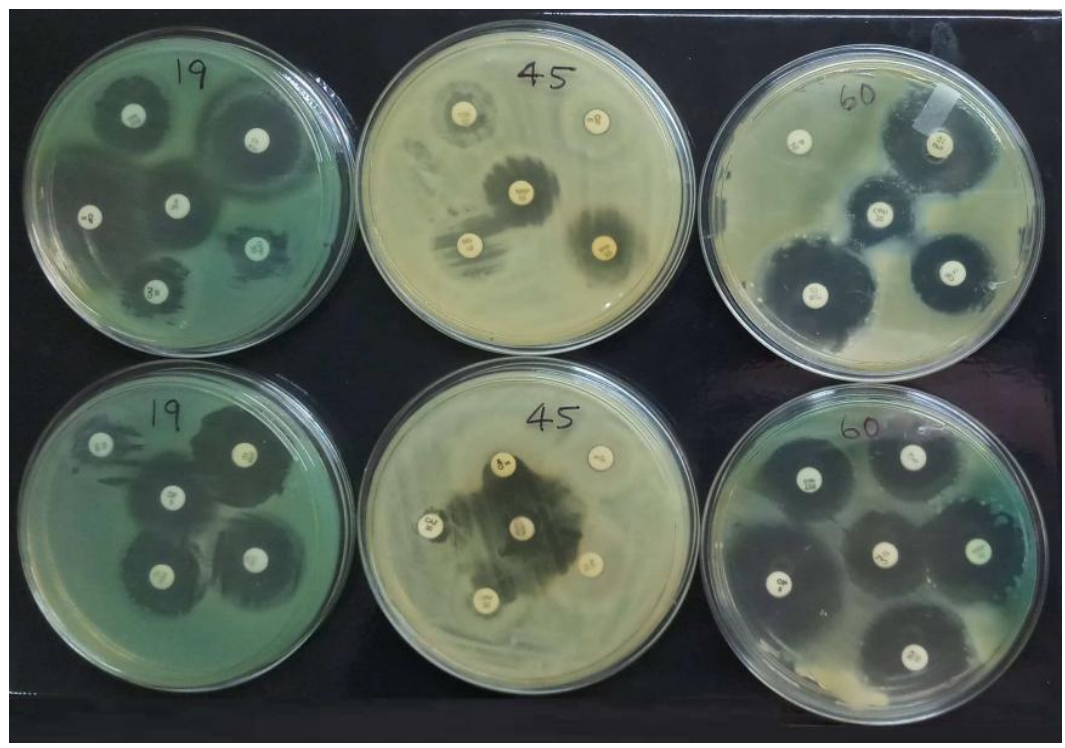

All the isolated multidrug resistant $P$. aeruginosa showed range of resistance from $36.84 \%$ to $100 \%$ of the 12 antibiotics tested. Resistance was reported against Penicillin (100\%), Oxacillin (100\%), Ampicillin (100\%), Vancomycin Cotrimoxazole (48.65\%), Tetracycline (78.94\%), Chloramphenicol (57.89\%), Erythromycin (52.63\%), Azithromycin (52.63\%), Streptomycin (47.36\%), Gentamycin (42.10\%) and Ciprofloxacin $(36.84 \%)$

The exposure of these drug resistant isolates is more challenging to clinical health concern. In this study, among isolates we found $51(50 \%)$ multidrug resistant strains show high level of infections of MDR P. aeruginosa. Over the past few decades, the extensive use of antibiotics leads to the increase in different multi drug resistant bacterial strains. Among isolated MDR strains, majority of strains showed resistance such as Piperacillin (98\%), Ciprofloxacin (90\%), Ofloxacin (90\%), Levofloxacin(80\%), Norfloxacin $(60 \%)$ and Tobramycin $(60 \%)$ and the lowest resistance was detected such as Ceftazidine, Cefepime, Piperacillin-tazobactam, Imipenem and Meropenem. Thus need regular study of MDR strain of Pseudomonas aeruginosa and its antibiotics sensitivity patterns for better treatment in future.

Pseudomonas aeruginosa is the most important agent causing nosocomial infections and it frequently develops resistance to the many antibiotics which is challenging for clinicians to treat so, this study desires to maintain proper hygienic condition of wards and ICU with proper hand hygiene maintains to prevent nosocomial infections of MDR strains of $P$. aeruginosa. The clinical samples were collected from VAMC and RH and processed in the laboratory andon the basis of their morphological characteristics and numerous biochemicals test $P$. aeruginosa were isolated. From these isolates MDR strains of $P$. aeruginosa identified according to antibiotic sensitivity pattern. These resistant strains indicate the improper use of antibiotics in the hospital. During study, $102 P$. aeruginosa strains were isolated from the 
clinical samples out of these the 51 MDR strains of $P$. aeruginosa were decleared on antibiogram pattern. We foundthe majority of strains showed resistance such as Piperacillin (98\%), Ciprofloxacin (90\%), Ofloxacin (90\%), Levofloxacin (80\%) and Tobramycin $(60 \%)$. So, in this study the MDR $P$. aeruginosa isolated these strains act as source for exploration the QscR protein. For controlling MDR strains of $P$. aeruginosa, we need to use alternative method that inhibiting the QS in $P$. aeruginosa by enhancing the transcriptional regulator.

\section{References}

1.C. P. Baveja, "Pseudononas, Stenotrophomonas and Burkholderia", Textbook of Microbiology, PP. 305, Sixth Edition: 2018.

2. R. F. Taylor, H. Gaya and M. E. Hodson, "Pseudomonas cepacia: Pulmonary infection in patients with cystic fibrosis", Respiratory Med. vol.87, pp.187-192, Apr. 1993.

3. M. Whiteley, K. M. Lee, and E. P. Greenberg, "Identification of genes controlled by quorum sensing in Pseudomonas aeruginosa", Proc. Natl. Acad. Sci., vol.96, pp. 13904-13909, Nov. 1999.

4. S. A. Chugani, M. Whiteley, K. M. Lee, D. D'Argenio, C. Manoil, and E. P. Greenberg, "QscR, a modulator of quorum sensing signal synthesis and virulence in Pseudomonas aeruginosa", Proc. Natl. Acad. Sci.vol.98, pp.2752-2757, Feb. 2001.

5. M. Gambello and B. H. Iglewski, "Cloning, characterization of the Pseudomonas aeruginosa lasR gene and a transcriptional activator of elastase production", J. Bacteriol, vol.173, pp.3000-3009, May.1991.

6. E. C. Pesci, J. P. Pearson, P. C. Seed, and B. H. Iglewski, "Regulation of las and rhl quorum sensing in Pseudomonas aeruginosa", J. Bacteriol., vol.179, pp.3127-3132, May 1997

7. M. R. Chandrashekar, K. C. Rathish and C. N. Nagesha, "Reservoirs of nosocomial pathogens in neonatal intensive care unit', Journal of the Indian Medical Association, vol.95, pp.72-74, Mar. 1997.

8. K. M. Gray, L. Passador, B. H. Iglewski, and E. P. Greenberg. Interchangeability and specificity of components from the quorum-sensing regulatory systems of Vibrio fischeri and Pseudomonas aeruginosa. J. Bacteriol, vol.176, pp.3076-3080, May 1994

9. A. M. Stevens, K. M. Dolan, and E. P. Greenberg, "Synergistic binding of the Vibrio fischeriLuxR transcriptional activator domain and RNA polymerase to the lux promoter region", Proc. Natl. Acad. Sci. vol. 91, pp.12619-12623. Dec. 1994

10. A. Latifi, M. Foglino, K. Tanaka, P. Williams, and A. Lazdunski, "A hierarchical quorum-sensing cascade in Pseudomonas aeruginosa links the transcriptional activators LasR and RhIR (VsmR) to expression of the stationary-phase sigma factor RpoS", Mol. Microbiol. vol.21, pp.1137-1146. Sep.1996

11. L. Passador, J. M. Cook, M. J. Gambello, L. Rust, and B. H. Iglewski, "Expression of Pseudomonas aeruginosa virulence genes requires cell-to-cell communication", Science, vol.260, pp.1127-1130.May. 1993

12. E. C. Pesci, J. B. J. Milbank and J. P. Pearson, "Quinolone signaling in the cell-to-cell communication system of Pseudomonas aeruginosa", ProcNatlAcadSci, vol.96, pp.1122911234. Sep. 1999

13. B. L. Bassler, E. P. Greenberg, and A. M. 
Stevens, "Cross species induction of luminescence in the quorum-sensing bacterium Vibrio harveyi", J. Bacteriol. vol.179, pp.4043-4045. Jun. 199

14. Y. H. Dong, J. L. Xu, X. Z. Li and L. H. Zhang, "AiiA, an enzyme that inactivates the acylhomoserine lactone quorum-sensing signal and attenuates the virulence of Erwiniacarotovora", Proceedings of the National Academy of Sciences, vol.97, pp.3526-31, Mar. 2000

15. E. C. Pesci, J. B. Milbank, J. P. Pearson, S. McKnight, A. S. Kende, E. P. Greenberg, and B.H. Iglewski, "Quinolone signaling in the cell-to-cell communication system of Pseudomonas aeruginosa", Proc. Natl. Acad. Sci. vol. 96, pp.122911234.Sep. 1999

16. M. R. Parsek and E. P. Greenberg, "Quorum sensing signals in development of Pseudomonas aeruginosa biofilms", Methods Enzymol.vol.310, pp.43-55, Jan.1999.

17. Japoni, A. S. Farshad and A. Alborzi, Pseudomonas aeruginosa Burn Infection, Treatment and Antibacterial Resistance, Iran Red Crescent Med J, 11(3), PP.244-253, 2009.

18. R. Moniri, Z. Mosayebi and Ah Movahedian, "Increasing trend of antimicrobial drug-resistance in Pseudomonas aeruginosa causing septisemia,' Iran J of Public Health, pp. 58-62, 35(1), May 2006.

19. D. A. A. Mossel, W. H. J. Mengerink, and H. H. Scholts, "Use of a modified macconkey agar medium for the selective growth and enumeration of enterobacteriaceae", Journal of Bacteriology, vol.84, pp.381.Aug. 1962.

20. J. G. Holt, N. R. Krieg, P. H. A. Sneath, J. T. Staley, and S. T. Williams,
"Bergey's Manual of Determinative Bacteriology" 9th Ed. Lippincott Williams and Wilkins. P-242. 1994

21. A. W. Bauer, M. D. K. Kirby, J. C. Sherris, and M. Turck, "Antibiotic susceptibility testing by standardized single disc diffusion method", Am J ClinPathol, vol.45, pp. 493-96. Apr. 1966

22. Clinical and Laboratory standards Institute, Performance standards for antimicrobial susceptibility testing; Seventeenth Informational Supplement. CLSI document M100S17. ISBN 1-56238-625-5.

23. R. E. Showalter, M. O. Martin, and M. R. Silverman, "Cloning and nucleotide sequence of luxR, a regulatory gene controlling bioluminescence in Vibrio harveyi", J. Bacteriol. vol. 172, pp. 2946-2954. Jun. 1990.

24. I. Biswal, B. S. Arora and N. Dimple Kasana, "Incidence of multidrug resistant Pseudomonas aeruginosa isolated from burn patients and environment ofteaching institution", Journal of clinical and diagnostic research: JCDR, vol.8, pp.DC26, May 2014

25. S. Shenoy, S. Baliga, D. R. Saldanha and H. V. Prashanth, "Antibiotic sensitivity patterns of Pseudomonas aeruginosa strains isolated from various clinical specimens", Indian J Med Sci, vol.56, pp.427-30. Sep. 2002

26. B. V. Ramana and A. Chaudhury, "Antibiotic resistance pattern of Pseudomonas aureuginosa isolated from healthcare associated infections at a tertiary care hospital", J SciSoc, vol.39, pp.78-80. May 2012.

27. C. Wattal, V. Kaul, T. D. Chugh, N. Kler and S. K. Bhandari, "An outbreak of multidrug resistant Salmonella typhimurium in Delhi', Indian J Med Res 100, Dec. 1994, pp.266-267. 
28. C. S. Swetha, A. Jagadeesh Babu, K. Venkateswara Rao, S. Bharathy, R. A. Supriya and T. Madhava Rao, “' Antimicrobial resistant pattern of Pseudomonas aeruginosa from raw milk samples" Asian J. Diary \& Food Res, 36(2)2017:100-105.
29. S. Shenoy, S. Baliga, D. R. Saldanha and H. V. Prashanth, "Antibiotic sensitivity patterns of Pseudomonas aeruginosa strains isolated from various clinical specimens", Indian journal of medical sciences, vol.56, pp.427-30. Sep. 2002.

\section{How to cite this article:}

Jitendra Kumar Chaudhary. 2021. A Clinical-Bacteriological Profile of Multidrug Resistant Pseudomonas aeruginosa at Tertiary Care Hospital Shahjahanpur, Uttar Pradesh. Int.J.Curr.Microbiol.App.Sci. 10(09): 304-313. doi: https://doi.org/10.20546/ijcmas.2021.1009.035 